\title{
Novel risk stratification and treatment
}

\author{
Takaaki Komatsu, Shiro Nakahara and Isao Taguchi \\ Hypertension Research (2016) 39, 574-575; doi:10.1038/hr.2016.39; published online 14 April 2016
}

\section{HYPERHOMOCYSTEINEMIA}

Most prospective studies of hyperhomocysteinemia have found an association with an increased risk of cardiovascular events. ${ }^{1}$ Several investigations have identified possible mechanisms for the development of atherosclerotic lesions following the elevation of this biomarker, including vascular endothelial defects, increased uptake of modified low-density lipoproteins (LDLs), stimulation of smooth muscle cell proliferation and defects of antithrombotic mechanisms. High levels of homocysteine are believed to promote the formation of oxidation products, such as homocystine, homocysteine disulfides and homocysteine thiolactone, which can damage endothelial cells through the excessive sulfuration of collagen. A clinical investigation found that for every unit $\left(\mu \mathrm{moll}{ }^{-1}\right)$ increase in the homocysteine level, a $7 \%$ increase in the risk of coronary artery disease (CAD) was observed, independent of traditional risk factors such as age, gender, body mass index, smoking, LDL cholesterol, family history of $\mathrm{CAD}$ and metabolic syndrome. ${ }^{2}$

Homocysteine is metabolized by two pathways: re-methylation and trans-sulfuration, both of which involve folic acid and B vitamins. Several studies have demonstrated that folate and B vitamins, either by supplementation or of dietary origin, can normalize low plasma homocysteine levels. However, reducing homocysteine levels by the consumption of $\mathrm{B}$ vitamins and/or folates did not lower cardiovascular risk. A few studies have speculated that this apparent inconsistency may be due to conflicting observational studies stemming from differences in genetic backgrounds. ${ }^{3}$ Despite the

T Komatsu, S Nakahara and I Taguchi are at Department of Cardiology, Dokkyo Medical University Koshigaya Hospital, Saitama, Japan

E-mail: taguchi@dokkyomed.ac.jp use of comprehensive measurements and adjustment strategies, the uncontrolled or residual differences in observational studies of dietary intake or supplementation are of concern. It is possible that populations with specific genetic backgrounds may benefit from folate or B vitamin supplements. Future clinical trials to evaluate the effects of such supplements in patients with hyperhomocysteinemia are needed in special subgroups to test these hypotheses.

\section{NEUTROPHIL-TO-LYMPHOCYTE RATIO}

The Neutrophil-to-lymphocyte ratio (NLR), a simple, readily available, inexpensive and noninvasive marker, has recently emerged as a more reliable indicator of inflammation, oxidative stress and atherosclerosis, and has been widely studied in several cardiovascular diseases. ${ }^{4}$ A recent important study by Shah et $a l .{ }^{5}$ demonstrated the contribution of the NLR to risk stratification and showed that the NLR significantly improves the prediction of $\mathrm{CAD}$ mortality over the Framingham Risk Score.

The mediation of an active inflammatory response by neutrophils is promoted by several biochemical pathways, such as the release of arachidonic acid metabolites and platelet-aggregating factors, cytotoxic oxygenderived free radicals and enzymes, such as myeloperoxidase, elastase, hydrolases and acid phosphatases. This low-grade and general systemic inflammatory response leads to neutrophilia and the relative lymphopenia of the cortisol-induced stress response.

A worsening of chronic kidney disease, which is a major risk factor of cardiovascular disease, has been associated with neutrophil activation. 6 ,7

Moreover, a high NLR may identify two further high-risk groups among hypertensive patients. A non-dipping blood pressure pattern has been associated with higher cardiovascular mortality, and patients with this pattern had significantly higher NLRs than patients with dipper hypertension. In addition, in the patients with obstructive sleep apnea, the NLR was high and positively correlated with the apnea-hypopnea index score. A value exceeding 1.62 was an independent predictor of obstructive sleep apnea.

However, NLRs have been reported to decrease after antihypertensive treatment with amlodipine and valsartan or with nebivolol. Therefore, NLR may also be a useful marker for treatment efficacy.

\section{NLR AND HOMOCYSTEINE}

Li et al. ${ }^{8}$ reported increased NLRs in subjects with both hypertension and hyperhomocysteinemia (HTH) and further reported that the NLR was positively correlated with homocysteine levels but not with blood pressure. A value of 1.88 or greater predicted $\mathrm{HTH}$ with a sensitivity of $45.2 \%$ and a specificity of $67.2 \%$. Li et al. ${ }^{8}$ also reported that patients with HTH had an increased risk of developing coronary atherosclerosis and vascular thrombosis in later life. The risk of cardiovascular events in individuals with $\mathrm{HTH}$ was nearly fivefold greater than those with simple hypertension, and 25-fold higher than those in healthy normotensive subjects. Therefore, the selection of patients with HTH by means of the NLR may be of great value in clinical settings because the NLR is a simpler and cheaper biomarker to determine than homocysteine.

However, the NLR is also associated with gender, age, glucose and creatinine levels, smoking, drinking and total plasma cholesterol. Furthermore, because the reference ranges for absolute neutrophil and lymphocyte counts are broad, a 'normal' NLR range is difficult to establish. Further studies are needed to explore whether NLR has a useful 


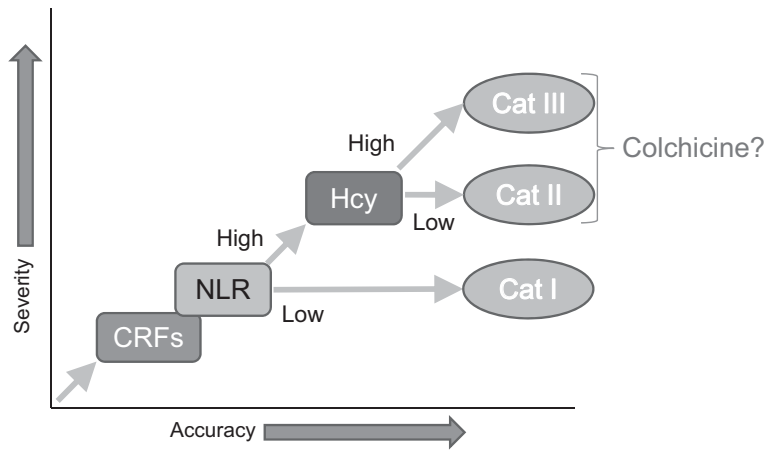

Figure 1 Proposed cardiovascular risk stratification with novel markers and treatment. Novel markers, such as NLR and Hcy, and the identification of CRFs, including hypertension, dyslipidemia, diabetes mellitus and smoking, may make risk stratification more accurate. The low-NLR group is Cat I; the high-NLR with low-Hcy group is Cat II; and the high-NLR and high-Hcy group is Cat III. Risk increases gradually from Cat I to Cat III. Colchicine could be recommended for the Cat II and III groups. Cat, category, CRFs, conventional risk factors; Hcy, homocysteine; NLR, neutrophil-to-lymphocyte ratio. A full color version of this figure is available at the Hypertension Research journal online.

threshold value that could accurately predict the incidence of cardiovascular events.

\section{COLCHICINE TREATMENT AND NOVEL RISK STRATIFICATION}

Although the renin-angiotensin system is a critical hormonal system associated with inflammation in cardiovascular diseases, ${ }^{9}$ colchicine is a long-standing drug with antiinflammatory effects, especially on neutrophils. Because neutrophils have critical roles in pericardial and atherosclerotic plaque inflammation, they represent a potential target for new drugs to treat and prevent cardiovascular diseases, such as pericarditis, atrial fibrillation in response to inflammation, and ischemic vascular events. Nidorf et al. ${ }^{10}$ reported that $0.5 \mathrm{mg}$ per day colchicine, administered in addition to statins and other standard secondary preventative therapies, appeared effective for the prevention of cardiovascular events in patients with stable coronary disease. Despite some caveats, colchicine may be an attractive therapy for the secondary prevention of cardiovascular events because it is simple to use, is inexpensive, appears to be highly effective and rarely leads to major irreversible toxicity during long-term use. However, before the drug is clinically adopted, the findings of the present hypothesis-generating study should be confirmed in larger studies of patients with coronary atherosclerosis.

The presence of activated neutrophils in the plaques of patients with unstable coronary syndromes has suggested that activated neutrophils may have a key role in the transformation of stable plaques to unstable plaques. ${ }^{11}$ Colchicine is known to have protean effects that may be responsible for the improved clinical outcome of patients with CAD through the inhibition of neutrophil chemotaxis, ingress and activation within a proinflammatory environment that may exist in an unstable plaque. Therefore, the NLR could be a useful marker for the selection of candidates for colchicine treatment.

Regarding the novel risk stratification presented in Figure 1, if the optimal cut-off value of the NLR was determined, when a patient has at least one conventional risk factor (for example, hypertension, diabetes mellitus, dyslipidemia, smoking and family history of CAD), the NLR should be calculated. If the NLR increases, serum homocysteine levels should be measured. Comprehensive cardiovascular risk could then be evaluated more accurately than with present methods.
In addition, a few patients with high NLRs may benefit from colchicine treatment.

\section{CONFLICT OF INTEREST}

The authors declare no conflict of interest.

1 Genest JJ, McNamara JR, Salem DN, Wilson PW, Schaefer EJ, Malinow MR. Plasma homocyst(e)ine levels in men with premature coronary artery disease. J Am Coll Cardiol 1990; 16: 1114-1119.

2 Hajer GR, van der Graaf Y, Olijhoek JK, Verhaar MC Visseren FL, SMART Study Group. Levels of homocysteine are increased in metabolic syndrome patients but are not associated with an increased cardiovascular risk, in contrast to patients without the metabolic syndrome. Heart 2007; 93: 216-220.

3 Bazzano LA, Reynolds K, Holder KN, He J. Effect of folic acid supplementation on risk of cardiovascular diseases: a meta-analysis of randomized controlled trials. JAMA 2006; 296: 2720-2726.

4 Horne BD, Anderson JL, John JM, Weaver A, Bair TL, Jensen KR, Renlund DG, Muhlestein JB, Intermountain Heart Collaborative Study Group. Which white blood cell subtypes predict increased cardiovascular risk? J Am Coll Cardiol 2005; 45: 1638-1643.

5 Shah N, Parikh V, Patel N, Patel N, Badheka A, Deshmukh A, Rathod A, Lafferty J. Neutrophil lymphocyte ratio significantly improves the Framingham risk score in prediction of coronary heart disease mortality: Insights from the National Health and Nutrition Examination Survey-III. Int J Cardiol 2014; 171: 390-397.

6 Heijnen BF, Van Essen H, Schalkwijk CG, Janssen BJ, Struijker-Boudier HA. Renal inflammatory markers during the onset of hypertension in spontaneously hypertensive rats. Hypertens Res 2014; 37: 100-109.

7 Hosohata K, Yoshioka D, Tanaka A, Ando $\mathrm{H}$ Fujimura A. Early urinary biomarkers for renal tubular damage in spontaneously hypertensive rats on a high salt intake. Hypertens Res 2016; 39: 19-26.

8 Li G, Zhang Y. Increased neutrophil to lymphocyte ratio in persons suffering from hypertension with hyperhomocysteinemia. Hypertens Res 2016; 39: 606-611.

9 Dhande I, Ma W, Hussain T. Angiotensin AT2 receptor stimulation is anti-inflammatory in lipopolysaccharide-activated THP-1 macrophages via increased interleukin-10 production. Hypertens Res 2015; 38: 21-29.

10 Nidorf SM, Eikelboom JW, Budgeon CA, Thompson PL. Low-dose colchicine for secondary prevention of cardiovascular disease. J Am Coll Cardiol 2013; 61: 404-410.

11 Naruko $T$, Ueda M, Haze $K$, Wal AC, van der Loos CM, Itoh A, Komatsu R, Ikura Y, Ogami M, Shimada Y, Ehara S, Yoshiyama M, Takeuchi K, Yoshikawa J, Becker AE. Neutrophil infiltration of culprit lesions in acute coronary syndromes. Circulation 2002; 106 : 2894-2900. 